\title{
Paisajes físico-geográficos de humedales costeros continentales en dos áreas naturales protegidas del Golfo de México ${ }^{\text {负 }}$
}

\section{Physical-geographic landscapes of two protected natural areas of coastal wetlands of the Gulf of Mexico}

\author{
Carlos Troche ${ }^{\mathrm{a}, \mathrm{b}}$, Ángel Guadalupe Priego-Santander*a ${ }^{*}$ Manuel Bollo Manent ${ }^{\mathrm{a}}$, Rainer Ressl ${ }^{\mathrm{b}}$ \\ ${ }^{a}$ Centro de Investigaciones en Geografía Ambiental (CIGA), Universidad Nacional Autónoma de México (UNAM). Campus Morelia. Antigua Carretera a \\ Pátzcuaro No. 8701, Ex-hacienda de San José de La Huerta, C.P. 58190, Morelia Michoacán, México \\ ${ }^{b}$ Comisión Nacional para el Conocimiento y Uso de la Biodiversidad (CONABIO), Av. Liga Periférico-Insurgentes Sur 4903, Col. Parques del Pedregal, Del. \\ Tlalpan, C.P. 14010, Ciudad de México, Mexico
}

\section{Resumen}

Los humedales costeros de Pantanos de Centla y Laguna de Términos en el Golfo de México, constituyen parte del complejo ecológico de la planicie costera más importante de Mesoamérica por su productividad natural. El objetivo del presente trabajo es el de clasificar y cartografiar, a escala 1:250 000, los paisajes naturales de estos humedales a partir del análisis de los componentes naturales del territorio. El enfoque utilizado para esta aproximación es el de la geografía física compleja que permite un arreglo tipológico y clasificación jerárquica del territorio. El mapa resultante presenta la distribución espacial de los paisajes físico-geográficos de la parte terrestre de la Reserva de la Biósfera Pantanos de Centla, del Área de Protección de Flora y Fauna Laguna de Términos y el sitio prioritario de manglar Sabancuy-Chen Kan de la Comisión Nacional para el Conocimiento y Uso de la Biodiversidad (CONABIO). La falta de cartografía precisa de los componentes naturales del territorio condicionó a un extenso trabajo de campo. En el área se definieron geocomplejos a nivel de Localidades, Parajes complejos y Parajes simples. En el territorio existe un predominio de los geocomplejos de planicies fluvio-biógenas. Los resultados son importantes para estudios de ordenamiento territorial y evaluaciones de servicios ecosistémicos.

Palabras clave: Paisaje; Ecosistemas costeros; Humedales; Pantanos de Centla; Laguna de Términos; México

\begin{abstract}
Due to their natural productivity, the coastal wetlands of "Pantanos de Centla" and "Laguna de Términos", in the Gulf of Mexico, form part of the ecological complex of the most important coastal plain of Mesoamerica. The objective of this work is to classify and map, at 1: 250000 scale, the natural landscapes of these wetlands applying analysis of the natural components of the territory. The approach is based on an approximation of the complex physical geography that allows a typological arrangement and hierarchical classification of the territory. The resulting map presents the spatial distribution of the physical-geographic landscapes of the terrestrial parts of the "Pantanos de Centla Biosphere Reserve", the "Laguna de Términos Flora and Fauna Protection Area" and the priority site of the National Commission for the Knowledge and Use of Biodiversity (CONABIO) "Sabancuy-Chen Kan". The lack of precise cartography of the natural components of the territory conditioned an extensive field work. In the area, geocomplexes were defined at the level of landscape, land area and sub-land area. As a result, we found, that in the territory there is a predominance of the geo-complexes of fluvio-biogenic plains. The results are important for land use studies and ecosystem service assessments.
\end{abstract}

Keywords: Landscape; Coastal ecosystems; Wetlands; Pantanos de Centla; Laguna de Términos; Mexico 


\section{Introducción}

En las últimas décadas se ha retomado la idea de que la delimitación de los espacios naturales en unidades homogéneas debe realizarse de manera holística. De esta forma se podrían entender mejor las propiedades, funciones y condiciones que estos sistemas presentan. También se podrían evaluar e identificar muchos de los servicios ambientales que estos brindan al ser humano (Priego-Santander et al., 2003; Termorshuizen \& Opdam, 2009). La ecología del paisaje, con sus diferentes escuelas, pretende ser ese instrumento integrador que permite, a partir de un enfoque interdisciplinario, la solución al enfrentarse a la heterogeneidad espacial de los paisajes. Una de las escuelas más antiguas, que es la Geografía Física Compleja o Geografía del Paisaje, basada en contribuciones de V.V. Dokuchaev y A. Humbolt (Mateo, 2002), tiene como metas principales el conocimiento de la génesis y estructura para la comprensión de la evolución y dinámica de los paisajes (Bocco et al., 2010). A partir de este enfoque, el arreglo geográfico de los paisajes está constituido por una tipología que permite clasificarlos por niveles jerárquicos, relacionados entre sí por parámetros o variables descriptivas esenciales de los paisajes (Priego-Santander et al., 2010).

Esta tipificación del paisaje, que permite la delimitación de unidades espaciales homogéneas, es útil para realizar diferentes tipos de análisis en el territorio. Muchos estudios utilizan este tipo de caracterización territorial para la planificación y manejo del paisaje (Palacio, 2006), predicción y relación con la biodiversidad (Priego-Santander et al., 2013), evaluaciones de degradación de los ambientes naturales (Mathews, 2008), entre otros. Los paisajes costeros, al constituirse en zonas terminales de las cuencas hidrográficas y encontrarse en zonas de transición entre la parte terrestre y marina, presentan una dinámica muy particular en comparación con otros ambientes. Es importante mencionar que para la evaluación de muchos procesos costeros es necesario, no solamente la delimitación espacial de unidades terrestres o marinas, sino también la definición de fronteras y delineación de unidades donde interactúan los componentes del ambiente costero (Palacio, 2006). En estos paisajes, generalmente planos, la parte hidrológica expresada en la periodicidad de inundación, se vuelve un factor de diferenciación ecológica muy importante (Priego-Santander et al., 2010). La región que comprende las áreas naturales protegidas de Pantanos de Centla y Laguna de Términos es de especial interés, desde el punto de vista físico-geográfico, por sus procesos costeros y la particularidad de sus sedimentos debido a dos causas principales: a) los procesos y tipos de sedimentación costera y; b) la

\footnotetext{
(C) C. Troche, A. G. Priego-Santander, M. Bollo Manent, Rainer Ressl. Published by Terra Digitalis.

This is an Open Access article distributed under the terms of the Creative Commons Attribution License (https://creativecommons.org/licenses/by-nc-sa/4.0/), which permits non-commencial sharing of the work and adaptions, provided the original work is properly cited and the new creations are licensed under identical terms.

*E-mail address: apriego@ciga. unam.mx
}

transición existente entre la plataforma de la Península de Yucatán, que aporta sedimentos de tipo carbonatado y la región de los ríos Grijalva-Usumacinta, Candelaria, Palizada, entre otros, que aportan con sus sedimentos de tipo fluvio-terrígenos (de distinta composición químico-mineralógica) a estos humedales costeros (Yáñez-Correa, 1971).

Como menciona Palacio (2006), en su estudio del paisaje costero de Laguna de Términos, existe una transformación constante de las fronteras de las unidades debido a la fluctuación rítmica de los niveles de marea, los regímenes de oleaje, corrientes de deriva litoral, así como las modificaciones en los aportes y distribución sedimentaria. Esto, aunado a los cambios y modificaciones en las coberturas naturales, nos indica que la actualización de los límites de las unidades de paisaje en estos ambientes es necesaria como base para cualquier tipo de evaluación.

El presente estudio tiene como objetivo el conocimiento de la distribución espacial y heterogeneidad físico-geográfica actual de una parte del complejo de humedales costeros más importante de Mesoamérica, presente en el Golfo de México, desde el punto de vista de biodiversidad, productividad y descarga de agua dulce y sedimentos. Este trabajo se realizó utilizando los límites de la Reserva de la Biósfera Pantanos de Centla (RBPC), el Área de Protección de Flora y Fauna Laguna de Términos (APFFLT) y el sitio prioritario de manglar "SabancuyChen Kan" de la Comisión Nacional para el Conocimiento y Uso de la biodiversidad (PY75-CONABIO) a una escala de 1:250 000 (ver Lámina 1).

La originalidad de esta investigación consiste en que, por vez primera, se logra el levantamiento, clasificación y cartografía de paisajes en humedales costeros tropicales de México a escala 1:250 000. El valor teórico del trabajo radica en el aporte de la taxonomía de ecosistemas a nivel geográfico para el área de estudio, pero su importancia práctica puede ser mucho mayor, pues se aportan las bases biofísicas para los procesos de ordenamiento ecológico del territorio y evaluaciones de servicios ecosistémicos.

\section{Métodos}

\section{1. Área de estudio}

El área de estudio se encuentra entre $17.96^{\circ}$ y $19.33^{\circ}$ de latitud norte y $92.80^{\circ}$ y $90.72^{\circ}$ de longitud oeste. Comprende la parte continental terrestre de la RBPC, APFFLT y el sitio prioritario PY75-CONABIO (CONABIO, 2009; CONANP, 2016). La parte marina y cuerpos de agua, no fueron clasificados tipológicamente debido a que el mapa de paisajes físico-geográficos fue elaborado para la evaluación de almacenes fijos de carbono de diferentes tipos de humedales en estos ecosistemas costeros. En total el área cartografiada y clasificada tipológicamente abarca una superficie de $9,050.6 \mathrm{~km}^{2}$, situados entre los estados de Tabasco y Campeche, México.

El clima costero de esta región es de tipo cálido sub-húmedo con lluvias en verano (García-CONABIO, 1998). La precipitación media anual va de $1,686 \mathrm{~mm}$ a $2,399 \mathrm{~mm}$, la temperatura 
media anual oscila entre 21.7 y $24.7^{\circ} \mathrm{C}$. Estos humedales costeros se caracterizan por presentar mayoritariamente planicies y suelos de drenaje deficiente tipo Gleysol, Fluvisol y Solonchak. En la cobertura vegetal encontramos comunidades de hidrófitas (Typha dominguensis, Cladium jamaicense, Cyperus sp., Pistia stratiotes, Pontederia sagittata, Phyllanthus fluitans, Nymphaea sp.), pastos (Spartina spartinae, Muhlenbergia sp.), selvas bajas, selvas medianas subperennifolias de inundación periódica (Manilkara zapota, Bursera sp.), vegetación riparia (Pachira aquatica, Bucida buceras), manglares (Rhizopora mangle, Languncularia racemosa, Avicennia germinans y Conocarpus erectus), matorral de mucal (Mimosa pigra) y algunos palmares inundables (Sabal mexicana) (INEGI, 2014; Lot, 2015).

\subsection{Mapeo de paisajes}

La delimitación y mapeo de las unidades tipológicas de paisaje se basaron en la definición de una estructura jerárquica con cuatro niveles taxonómicos (Tabla 1), fundamentada en los postulados de la Geografía Física Compleja, referidos por Mateo (2002), adaptados y usados ampliamente en el contexto geográfico mexicano (Priego et al., 2016; Morales et al., 2017).

Tabla 1: Definición y criterios de las unidades taxonómicas Table 1. Taxonomic units definition and criteria

\begin{tabular}{cl}
\hline Nivel taxonómico & Índices diagnóstico \\
\hline Clase & Conjunto de morfo-estructuras con \\
& tipo específico de relieve en una \\
& misma condición climática. \\
& Comunidad territorial de igual tipo \\
& morfogenético de relieve. Presenta \\
& similar homogeneidad litológica o \\
& tipo de depósitos. \\
& Asociaciones del mismo conjunto \\
& morfológico de mesoformas del re- \\
& lieve. Predominio de iguales agru- \\
& pamientos de suelos y similar con- \\
& junto de formaciones vegetales y ti- \\
& pos de suelo. \\
& Misma periodicidad de inundación \\
& con mismo tipo de comunidades \\
& vegetales y similares grupos y sub- \\
& grupos de suelo. \\
\hline
\end{tabular}

La elaboración del mapa de los paisajes físico-geográficos del área de estudio a escala 1: 250 000, siguió la propuesta de generación de unidades de paisajes, de manera semi-automatizada de Priego et al. (2010), con algunas modificaciones de acuerdo a las características del área de estudio. Los insumos cartográficos utilizados para realización del mapa de paisajes fueron los siguientes:

- Mapas topográficos digitales de Campeche y Tabasco a escala 1:50 000 (INEGI, 2016).

- Mapa digital de los climas de México a escala 1:1000 000 (García-CONABIO, 1998).
- Mapas geológicos digitales de Campeche y Tabasco a escala 1:250 000 (INEGI, 1981; SGM, 2004, 2005)

- Mapa digital edafológico de México a escala 1:250 000 (INEGI, 2013)

- Mapa de uso de suelo y vegetación de la zona costera a escala 1:50 000 (CONABIO, 2016)

- Mapa digital de áreas de inundación de la Laguna de Términos a escala 1:250 000 (EPOMEX-IG/UNAM-CONABIO, 2000)

Cada mapa fue homogenizado para que su extensión y límites coincidieran entre sí, utilizando como marco de referencia el mapa de uso de suelo y vegetación de la zona costera de la CONABIO (2016). Las etapas para determinar cada nivel taxonómico, según la definición y criterios descritos en la Tabla 1, fueron las siguientes:

\section{Nivel Taxonómico: Clase.}

A partir de las curvas de nivel, se generó el modelo digital de elevación con el cual se determinó que morfométricamente el área de estudio se encuentra en planicies costeras sub-horizontales, según su disección vertical ( $\left.\mathrm{Dv} \leq 2.5 \mathrm{~m} / \mathrm{km}^{2}\right)$. Posteriormente se realizó la definición climática de estas planicies con la sobreposición de tipos de clima, cuyo resultado fue de planicies en clima cálido para toda el área. En este punto de la integración del tipo de clima, es necesario realizar las siguientes consideraciones según las premisas de Priego et al. (2010): a) Aunque el mapa de clima presenta una escala de 1: 1000000 , fue utilizado para este producto de 1:250 000, ya que es la única fuente oficial de climas en el país; b) el clima posee una distribución continua y en la naturaleza la diferenciación entre tipos de clima se realiza de una forma gradual y continua. En excepciones los cambios son abruptos; c) Los tipos de clima ocupan extensas áreas, lo que reduce los errores que se pueden cometer al ampliar un mapa 1:1 000000 a escalas mayores; d) el área de estudio comprende un solo tipo climático (cálido húmedo), lo que facilita la determinación de esta clase.

\section{Nivel Taxonómico: Localidad.}

La base de este nivel taxonómico estuvo centrada en la determinación de la composición litológica de estas planicies, a partir de la sobreposición de los mapas geológicos del Servicio Geológico Mexicano (SGM) y del Instituto Nacional de Estadística y Geografía (INEGI). Se determinaron los tipos litológicos consistentes con la zona y se eliminaron todos los polígonos que no cumplieran con el área mínima cartografiable $\left(1 \mathrm{~km}^{2}\right)$. El trabajo de campo para validación y/o corrección de las unidades superiores del mapa y la leyenda, así como la obtención de 
información de vegetación y periodicidad de inundación, fueron útiles y muy importantes para la conformación de las unidades intermedias e inferiores.

3. Nivel Taxonómico: Paraje complejo.

Al resultado anterior, se sobrepuso cartográficamente el mapa de uso de suelo y vegetación y posteriormente el mapa edafológico, teniendo como resultado la integración de estas dos capas (vegetación y suelos). De esta manera se generaron las unidades intermedias de paisajes o nivel de Paraje complejo. Posteriormente, se realizó la generalización conceptual y espacial del producto (aplicación del área mínima cartografiable de $1 \mathrm{~km}^{2}$ ) y se determinó el grupo de vegetación presente en cada unidad de paisaje.

\section{Nivel Taxonómico: Paraje simple.}

Para este nivel, las unidades se desagregaron por tipo de vegetación y periodicidad de inundación, obtenida a partir de datos de campo, vegetación y mapas de inundación del área de estudio. Como mencionan Priego et al. (2010), la periodicidad de inundación es un factor ecológico importante de diferenciación. Estas unidades, según su periodicidad, pueden llegar a ser muy dependientes de la interacción con los usos del territorio adyacente y el gradiente de inundación puede permitir el establecimiento de un continuo entre sistemas lago-humedal-tierra. Por lo anterior, se aplicó la clasificación basada en el periodo de meses en que el suelo está saturado en agua.

Para la representación cartográfica se empleó el método del fondo cualitativo para las Localidades (Lámina 1. La leyenda ampliada puede consultarse en la sección: Material suplementario) y símbolos numéricos para los Parajes simples. Los Parajes complejos se representan indirectamente, a través de sus unidades inferiores.

\section{Conclusiones}

Este estudio presenta una metodología para la identificación espacial de unidades naturales del paisaje en humedales costeros, en el que la génesis del relieve y la periodicidad de inundación son criterios importantes en la diferenciación de unidades inferiores.

Como limitaciones de la investigación, se puede señalar la falta de información cartográfica precisa sobre los componentes naturales del territorio, lo cual condicionó un intenso trabajo de campo. Esta falta de información precisa, parece ser una peculiaridad de los humedales costeros en gran parte del territorio nacional.

En áreas tan extensas de humedales costeros, el trabajo de campo se convierte en algo fundamental para eliminar incon- gruencias cartográficas encontradas al momento de la integración de los diferentes atributos del territorio. Por otro lado, también sirve para la verificación y validación de la hipótesis cartográfica realizada.

En el área de estudio se pudieron identificar tres niveles taxonómicos de nivel local; i) 6 localidades; ii) 11 parajes complejos y iii) 61 parajes simples.

Las planicies fluvio-biógenas acumulativas presentan el mayor porcentaje de superficie con $48 \%$ del total del área de estudio. Le siguen las planicies fluviales acumulativas con $25 \%$, las planicies marino-terrígenas acumulativas con $11 \%$, las planicies marino-eólicas acumulativas con $7 \%$, las planicies fluviotectónicas con $5 \%$ y las planicies acumulativas con $4 \%$. De las dos áreas naturales protegidas, la Reserva de la Biósfera Pantanos de Centla presenta tres Localidades con fuerte predominio de la dinámica fluvial. El $58 \%$ de esta reserva presenta planicies fluvio-tectónicas acumulativas, formadas por depósitos aluviales y calizas en clima cálido húmedo. Posteriormente, se encuentran las planicies fluviales acumulativas, formadas por depósitos aluviales en clima cálido húmedo con $36 \%$ y el restante $6 \%$ se compone de planicies tectónicas abrasivo-acumulativas, formadas por calizas en clima cálido húmedo.

En el Área de Protección de Flora y Fauna Laguna de Términos se encuentran representadas 5 localidades. Al igual que en Pantanos de Centla, las planicies fluvio-biógenas acumulativas y las planicies fluviales se encuentran ampliamente distribuidas con $61 \%$ y $11 \%$ respectivamente. Las planicies marinoeólicas se encuentran en $9 \%$ del territorio. Finalmente, el entendimiento de la composición y estructura físico-geográfica del área de Pantanos de Centla y Laguna de Términos, así como la comprensión de su heterogeneidad podrá ayudar a realizar evaluaciones espaciales integrales. Estas evaluaciones son útiles para sentar las bases biofísicas en procesos de ordenamiento ecológico del territorio, así como evaluaciones de diferentes tipos de servicios que estos ecosistemas nos brindan.

\section{Software}

La integración, generalización y composición del mapa se llevó a cabo usando Esri ArcGis 10.3.1. Los metadatos se pueden consultar en la sección: Material suplementario.

\section{Agradecimientos}

Este estudio fue financiado por la Comisión Nacional para el Conocimiento y Uso de la Biodiversidad (CONABIO), así como el posgrado de Geografía de la Universidad Nacional Autónoma de México (UNAM). Queremos agradecer en particular al Dr. José Sarukhán por el apoyo incondicional del fortalecimiento de capacidades institucionales. También agradecemos a la sub-coordinación de Percepción Remota de la CONABIO por el uso de sus instalaciones. A la Comisión Nacional de Áreas Naturales Protegidas (CONANP) por el apoyo en campo y logístico brindado en la Reserva de la Biósfera Pantanos de Centla y Reserva de Flora y Fauna Laguna de Términos. 
PAISAJES FÍSICO-GEOGRÁFICOS DE HUMEDALES COSTEROS CONTINENTALES EN DOS ÁREAS NATURALES PROTEGIDAS DEL GOLFO DE MÉXICO, MÉXICO 1:250 000 I PHYSICAL-GEOGRAPHIC LANDSCAPES OF TWO PROTECTED NATURAL AREAS OF COASTAL WETLANDS OF THE GULF OF MEXICO, MEXICO 1:250 000 Autores /Autors: Troche, C., Priego-Santander, A. G., Bollo Manent, M., Ressl, R.

"Centro de Investigaciones en Geografía Ambiental (CIGA), Universidad Nacional Autónoma de México (UNAM)
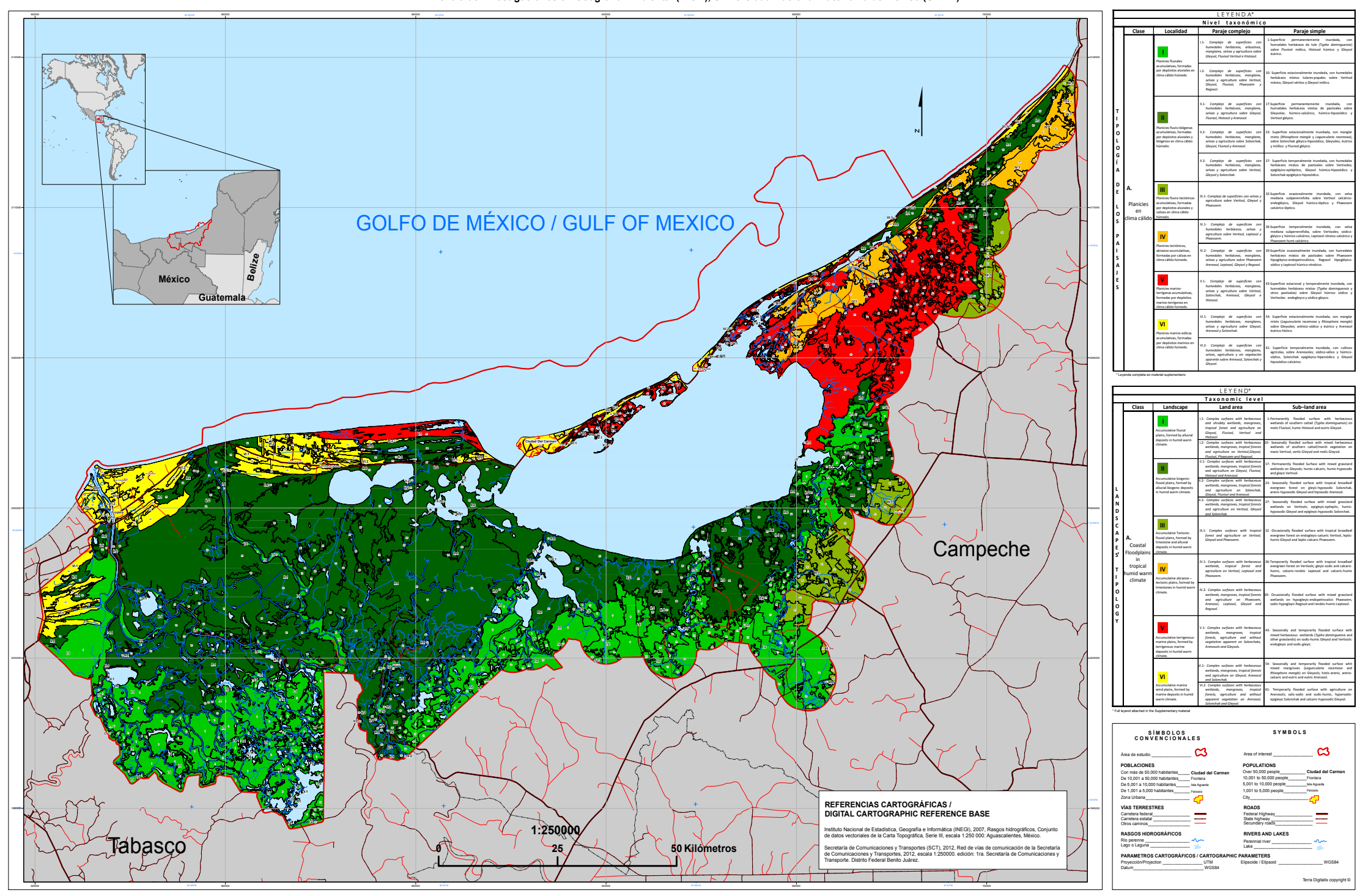


\section{Referencias}

Bocco, G., Mendoza, M., Priego, A., Burgos, A., 2010. La cartografía de los sistemas naturales como base geográfica para la planeación territorial: Una revisión bibliográfica. Secretaría de Medio Ambiente y Recursos Naturales, Instituto Nacional de Ecología, Universidad Nacional Autónoma de México, Centro de Investigaciones en Geografía Ambiental. México. 72 pp.

Centro EPOMEX, Instituto de Geografía-UNAM, CONABIO, 2000. Áreas de inundación de la Laguna de Términos, Campeche: Extraído del proyecto N011, Actualización del Mapa de Uso del Suelo, Vegetación y Hábitats Críticos y Elaboración de una Base Cartográfica Digital del Área Protegida de la Laguna de Términos. Escala 1:250 000. Centro EPOMEX, Instituto de Geografía-UNAM, CONABIO. México. D.F.

Comisión Nacional de Áreas Naturales Protegidas (CONANP), 2016. Áreas Naturales Protegidas Federales de la República Mexicana, (en línea), $<$ http://sig.conanp.gob.mx/website/pagsig/>, consulta: 18 de agosto de 2017.

Comisión Nacional para el Conocimiento y Uso de la Biodiversidad (CONABIO), 2009. Sitios de manglar con relevancia biológica y con necesidades de rehabilitación ecológica, (en línea), $<$ http://www.conabio.gob.mx/informacion/gis/>, consulta: 6 de octubre de 2017.

Comisión Nacional para el Conocimiento y Uso de la Biodiversidad (CONABIO), 2016. Mapa de uso del suelo y vegetación de la zona costera asociada a los manglares de México en 2015, escala: 1:50 000: (en línea), $<$ http://www.conabio.gob.mx/informacion/gis/>, consulta: 16 de diciembre de 2016.

García, E., Comisión Nacional para el Conocimiento y Uso de la Biodiversidad (CONABIO), 1998. Climas (clasificación de Koppen, modificado por García). Escala 1:1 000 000. México.

Instituto Nacional de Estadística, Geografía e Información (INEGI), 1981. Conjunto de Datos Geológicos Vectoriales, cartas E1503, E1505, E1506, E1508 a Escala 1:250000. Serie I, Aguascalientes, Ags., México, (en línea), $<$ http://www.inegi.org.mx/geo/contenidos/recnat/geologia/Default.aspx $>$, consulta: 8 de junio, 2016

Instituto Nacional de Estadística, Geografía e Información (INEGI), 2013. Conjunto de Datos Vectorial Edafológico, cartas E1503, E1505, E1506, E1508 a Escala 1:250000, Serie II, Aguascalientes, México, (en línea), $<$ http://www.inegi.org.mx/geo/contenidos/recnat/edafologia/Default.aspx>, consulta: 14 de julio, 2016.

Instituto Nacional de Estadística, Geografía e Información (INEGI), 2014. Guía para la interpretación de cartografía: uso del suelo y vegetación: escala 1:250 000: serie V / Instituto Nacional de Estadística y Geografía. México, (en línea), <http://www.inegi.org.mx/geo/contenidos/recnat/default.aspx>, consulta: 03 de octubre, 2017.

Instituto Nacional de Estadística, Geografía e Información (INEGI), 2016. Conjunto de Datos Vectoriales de información topográfica, cartas E15B37, E15B46, E15B47, E15B55, E15B56, E15B57, E15B61, E15B62, E15B64, E15B65, E15B71, E15B72, E15B73, E15B74, E15B75, E15B76, E15B81, E15B82, E15B83, E15B84, E15B85, E15B86, E15D12, E15D13 a Escala 1:50 000, Serie III, Aguascalientes, México, (en línea),

$<$ http://www.inegi.org.mx/geo/contenidos/topografia/default.aspx $>$ consulta: 10 de octubre, 2016

Lot, A., 2015. Catálogo de la flora y vegetación de los humedales mexicanos. Universidad Nacional Autónoma de México, México D.F., 172 pp.

Mateo, J., 2002. Geoecología de los Paisajes: Bases para la Planificación y Gestión Ambiental. Universidad de La Habana, MES, Cuba, 205 pp.

Mathews, J., 2008. Evaluación de la modificación edafo-biógena de los Paisajes de la Región Sierra-Costa de Michoacán, México. Tesis de maestría en Geografía. Universidad Nacional Autónoma de México, Morelia-Michoacán, 64 pp.

Morales, H., Priego-Santander, A., Bollo, M., 2017. Los paisajes físicogeográficos del estado de Chiapas, México a escala 1:250 000. Terra Digitalis 1(1), 1-7, doi:10.22201/igg.terradigitalis.2017.1.8.71.

Palacio, A. G., 2006. Ecología del Paisaje costero en Laguna de Términos, Campeche. Diagnóstico Ambiental para el Manejo. Tesis doctoral. Universidad Nacional Autónoma de México, México, D.F., 136 pp.

Priego-Santander, A. G., Bocco, G., Mendoza, M., Garrido, A., 2010. Propuesta para la Generación Semiautomatizada de Unidades de Paisaje. Fundamentos y Métodos. Serie Planificación Territorial, Semarnat-INE-CIGA, 104 pp.

Priego-Santander, A. G., Bocco-Verdinelli, G., 2016. Paisajes Naturales. En Moncada-Moya, O. y López-López, A. (Coordinadores): Geografía de México. Una reflexión espacial contemporánea, Tomo I. Universidad Nacional Autónoma de México, 375 p.

Priego-Santander, A. G., Campos, M., Bocco, G., Ramírez-Sanchez, L. G., 2013. Relationship between landscape heterogeneity and plant species richness in the mexican pacific coast. Applied Geography 40, 171-178.

Priego-Santander, A. G., Moreno-Casadola, P., Palacio-Prieto, J. L., LópezPortillo, J., Geissert-Kientz, D., 2003. Relación entre la heterogeneidad del paisaje y la riqueza de especies de flora en cuencas costeras del estado de Veracruz, México. Investigaciones Geográficas 52, 31-52.

Servicio Geológico Mexicano (SGM), 2004. Carta Geológica-Minera Frontera E15-5, Tabasco, Campeche., Esc. 1:250,000. Serie I. Pachuca, Hidalgo, México.

Servicio Geológico Mexicano (SGM), 2005. Carta Geológica-Minera Ciudad del Carmen E15-6, Campeche E15-3, Villahermosa E15-8, Esc. 1:250,000. Serie I, Pachuca, Hidalgo, México.

Termorshuizen, J., Opdam, P., 2009. Landscape services as a bridge between landscape ecology and sustainable development. Landscape Ecology 24 1037-1052, doi:10.1007/s10980-008-9314-8.

Yañez-Correa, A., 1971. Procesos costeros y sedimentos recientes de la plataforma continental al sur de la Bahía de Campeche. Boletín de la Sociedad Geológica Mexicana 32 (2), 75-115.

\section{This article accompanies the following material:}

HTML:

Static map:

Interactive map: Supplementary Material 2: DOI: 10.22201/igg.terradigitalis.2018.1.41.91 